\title{
Choice of grafts or prostheses for valvular replacement
}

\author{
Dwight C. McGoon \\ From the Mayo Clinic and Mayo Foundation Section of Thoracic and Cardiovascular Surgery, \\ Rochester, Minnesota, U.S.A.
}

An attempt has been made to determine the important criteria for evaluation of various substitute valves from the standpoint of practical clinical experience, and to rate the broadly grouped types of valves with respect to these criteria. Stented fascia lata valves received the highest overall ranking, but, owing to their recent introduction, should probably not be generally adopted by most surgeons in preference to second and third prosthetic or homograft valves until a favourable record is proved by another two to three years of experience.

Concern and uncertainty are the prevalent attitudes to selection of the proper substitute valve for insertion after excision of diseased cardiac valves. The vantage provided by a completed decade of valvular replacement discloses a monumental record of intense activity which has had the general movement of progress. The vast number of types and models of prostheses and tissue valves which have been constructed, tested, and inserted in patients taxes the credulity of even observers close to the field, for if a comprehensive list of the various different and evolving types could be compiled it would number several hundreds.

In spite of the resulting uncertainty, the surgeon is forced to make a decision by the time the diseased valve of his patient has been excised and the field prepared for the substitute valve. A choice at the time is inescapable. Fortunately, the record of prosthetic valves and tissue valves has been generally good, and each offers the symptomatic patient with serious cardiac valvular disease a considerably improved outlook over that which would be his plight if operation were unavailable. It is appropriate to review and weigh the criteria which should be applied to the selection of the best valve and to attempt some tentative conclusions.

The proponents of the various types of prostheses and tissue valves naturally stress the importance of those very factors for which their particular valve is most outstanding. Study of the problem through these sources, therefore, can be as bewildering as the selection of the best automobile or electrical appliance on the market. Though no individual, and certainly not this author, can be suffici- ently authoritative to present a 'consumer's guide' in the field of valvular replacement, nevertheless the method of approach and tentative conclusions drawn by one surgeon may be of some interest and value.

\section{General orientation}

Although new valves and methods must be conceived, developed, and tested, the field in general has advanced to a degree of reliability which contradicts the widespread adoption of a new valve before at least a baseline of results which extend over a test period of two to five years or more can be presented by its proponents. Stated differently, we should be reluctant to abandon old techniques which have been tried and tested for several years and which have given reasonably good results, for new methods which may be theoretically attractive in several areas but which may in application prove to have unexpected deficiencies which outweigh the anticipated advantages. For the same reasons the time is approaching, or has arrived, when the arbitrary testing of new valve substitutes in humans without extensive preliminary evaluation in animals should be discouraged.

Another straightforward generality should be stated - namely, that the preferred operation is the one which enables the patient to remain alive and well for the longest time. Therefore the selection of the optimal substitute valve requires information concerning the late results after insertion of the various available valves. Such information is most meaningful when it includes percentages of patients alive and well in terms of elapsed time since operation. When follow-up periods of variable length are involved, only time- 
TABLE Comparison of substitute valves

\begin{tabular}{|c|c|c|c|c|c|c|}
\hline & Prostheses & $\begin{array}{l}\text { Homogra } \\
\text { Fresh }\end{array}$ & $\begin{array}{l}\text { afts } \\
\text { Preserved }\end{array}$ & Heterografts & $\begin{array}{l}\text { Pulmonary } \\
\text { autograft }\end{array}$ & $\begin{array}{l}\text { Stented autogenous } \\
\text { fascia lata }\end{array}$ \\
\hline $\begin{array}{l}\text { Durability } \\
\text { Haemodynamic characteristics } \\
\text { Thromboembolism } \\
\text { Assurance of function } \\
\text { Availability and choice of size } \\
\text { Ease of insertion } \\
\text { Record of survival }\end{array}$ & $\begin{array}{l}++ \\
+ \\
+ \\
++ \\
+++ \\
+++ \\
++\end{array}$ & $\begin{array}{l}++\frac{1}{2} \\
+++ \\
+++ \\
+ \\
0 \\
+(++) \\
++\end{array}$ & $\begin{array}{l}++ \\
+++ \\
+++ \\
+ \\
+ \\
+(++) \\
+\end{array}$ & $\begin{array}{l}+ \\
+++ \\
+++ \\
+ \\
++ \\
+(++) \\
0\end{array}$ & $\begin{array}{l}++\frac{1}{2} \\
+++ \\
+++ \\
+ \\
0 \\
+ \\
+\end{array}$ & $\begin{array}{l}++ \\
+++ \\
+++ \\
++ \\
++ \\
++ \\
+\end{array}$ \\
\hline Total & 14 & 13 & $12 \frac{1}{2}$ & II $\frac{1}{2}$ & II $\frac{1}{2}$ & 15 \\
\hline
\end{tabular}

$0=$ unfavourable $+=$ fair $;++=$ good $;+++=$ excell $3 n t$.

oriented data, such as actuarial curves, allow accurate comparison.

\section{Criteria for comparison (see Table)}

Because any valve substitute must qualify to a basic minimum of requirements it becomes difficult to state which is the most important or which are the major and which the minor characteristics for comparison of the various substitute valves. However, durability, haemodynamic characteristics, and the record of survival after implantation are of major importance. By durability is meant the capability of the substitute valve to maintain its normal features and function over many years, and preferably decades, of use. Haemodynamic characteristics refer to the flow pathways and gradients associated with a particular substitute valve, using the haemodynamic characteristics of the normal valve in its natural state as a reference for the ideal. The record of survival, of course, refers to the percentage of comparable patients alive and well for comparable intervals of time following insertion of the various valves. Other important criteria are the propensity to develop thromboembolism and hence the requirement for anticoagulation with its attendant disadvantages. Though a substitute valve may have satisfactory haemodynamic characteristics under ideal conditions, there may be variability in assurance of normal function after insertion owing to technical problems of proper positioning and suturing. In actual clinical application the availability and choice of size of substitute valves assume significant importance. Likewise, the ease with which a given valve can be inserted has a bearing on the risk of the operation itself and the suitability for employment of that valve by surgeons around the world who may not have acquired the particular insight and skill required for placement of certain types of substitute valves. Also pertinent is the question of the propensity of a given valve to develop infection, but since there is little discernible difference in this complication among the various types of substitute valves it is not separately considered in the present analysis.

\section{Comparison of criteria and type of sub- stitute valve}

The types of substitute valves can be broadly classified into prosthetic or tissue valves. The prosthetic valves can be further subdivided into lateral flow or central flow types, and the tissue valves into homografts of the fresh and preserved types, heterografts, and autografts, either of autogenous pulmonary valve or stented autogenous fascia lata. Because of the vast number of prostheses available, and because to some extent they share common characteristics, they have all been grouped for this comparison into one category. The Table lists the evaluation or grade given by this single observer to each of the criteria for each of the types of substitute valves.

Durability The prosthetic valves, particularly the ball valve prosthesis, have had an excellent record of durability, with the exception of the development of ball variance at an incidence which varies from low to high in the various series but which has so far been limited to the initial models of prostheses. Its occurrence may now be minimized by alterations in curing and preparation of the silicone rubber and also by avoidance of the use of silicone rubber in the newer types of clothcovered prostheses. It appears that the least satisfactory record of durability has been with the heterograft valve as prepared by formalin fixation, and that the best record has been achieved by the fresh homograft and autograft valves. Durability is one of the outstanding theoretical advantages of the stented fascia lata valve, but since inadequate time has elapsed to allow confirmation of this theo- 
retical advantage only a modest grade was thought advisable at this time.

Haemodynamic characteristics Since the natural is considered the ideal, the tissue valves, which allow central flow and the natural semilunar cusp configuration, all receive a top grade. In this evaluation the grade given to the prosthetic group may be too severe, since in actual practice transvalvular gradients have not differed significantly between the prosthetic and the tissue valve. However, turbulence is more severe, and the presence of the poppet directly in the pathway of blood flow is of concern. It is in this area that the newer tilting disc prostheses have their greatest appeal.

Thrombo-embolism Here again the tissue valves have their clearest advantage over the prosthetic valves, for this complication is most unusual after placement of each of the tissue valves. Perhaps the disadvantage held by the prostheses owing to the propensity of thromboembolism can be overcome. It is clear that this threat has been reduced by the improved evolving design of the prostheses and probably by 'encapsulation' through use of completely cloth-covered prostheses.

Assurance of function One of the greatest disappointments encountered by cardiac surgeons is the discovery of improper valvular function, usually insufficiency, either early or late after valvular replacement. The only requirement to prevent this complication after use of a prosthesis or a perfectly prepared stented valve is that the prosthesis be accurately sutured to the annulus of the resected valve. If the annulus has been carefully decalcified and prepared, and if sufficient sutures are well placed and well tied, such malfunction should be rare. However, in the case of unstented tissue valves, in addition to the above requirements, the valve must be precisely attached so that the commissures are ideally positioned and supported. This may not be readily accomplished, particularly when there is disproportion between the size and configuration of the recipient annulus or where the surgeon is relatively inexperienced in this technique. Therefore a lower grade is warranted in this category for the unstented types of tissue valve.

Availability and choice of size One of the chief advantages of prosthetic valves is that they are available in a wide range of sizes, the only requirement being a financial one, which hopefully can be minimized if stan- dardization and refinements in production can evolve. The most severe restriction in the use of fresh homograft valves is availability. Heterografts have the advantage over preserved homografts in that they can be harvested and prepared with very little more complexity than the manufacture of a prosthetic valve. Though each patient possesses a pulmonary autograft available for use, the extensive surgical requirement for its procurement and replacement is a strongly negative characteristic of this type of substitute valve. Fascia lata is likewise available in essentially all individuals but does require an additional incision and dissection, and the valve is constructed during the operative procedure itself.

Ease of insertion It is true that this criterion is closely related to that of assurance of function, and this analysis could be criticized for allowing the advantage of prosthetic valves in this regard to be awarded twice. However, I have retained the criterion separately because of its distinct meaning. It is of interest that in most reported series where tissue valves have failed and a secondary operation is required, or where coronary artery disease, advanced age, or other complicating features are present, surgeons who in other circumstances would have used a tissue valve prefer to use a prosthetic valve. This must be related to the ease and rapidity with which the prosthetic valve can be inserted in comparison with the tissue valve, and is a factor of pragmatic importance when considering the widespread performance of valvular replacement in centres large and small in all parts of the world. The grade in parentheses given to certain of the tissue valves refers to those which are placed in the mitral position and which are stented and therefore approach the prosthetic valve with respect to technical ease of insertion (arbitrarily, for purposes of summation, a grade of $\mathrm{I} \frac{1}{2}+$ is given to these types of valves).

Record of survival The ultimate criterion would, of course, be the actual record of survival in various groups if they could be made strictly comparable in surgical technique, case selection, and time elapsed since operation. A broad averaging of the reports available which provide sufficient data for reasonable comparison suggests that the formalin preserved heterografts have given the least satisfactory long-term result, with the fresh homograft and prosthetic valves maintaining a comparable late result. In series of cases at institutions where tissue valves are inserted in the more ideally selected patients and prosthetic 
valves are used in poor risk cases, comparative evaluations are biased against the results achieved in prosthetic valves. Nevertheless, it is the eight years of hard factual data available for the prosthetic valve, particularly the ball valve prosthesis, that is one of the strongest advantages enjoyed by the prosthetic group and which makes many surgeons, including the author, reluctant to abandon its use. The autograft valves can be graded only tentatively and with difficulty because of the short time elapsed since their introduction, and with further time their indicated grade in this category may be significantly improved.

\section{Summation of comparison}

Because of varying importance of the various criteria evaluated, simple addition of the grades given in the several categories may imply inaccurate conclusions. However, by doing so it is evident that the span between the lowest and highest total grade is not great. The valve receiving the highest total is the stented autogenous fascia lata, which on the one hand must be taken with reservation because of the recent introduction of this technique and the possibility that its durability is much less satisfactory in practice than in theory. On the other hand, further time may also show that this optimism is warranted and that the long-term record in actual application of the technique is better than estimated here. Next highest is the prosthetic group (based particularly on the ball valve prosthesis), with one of its strongest advantages being the large number of valves inserted and the several years elapsed since its initial use. The lowest ranking was given the heterograft group of valves, but it must be recognized that formalin fixation of such valves may not have been the best choice, and that application to this use of the knowledge gained through the science of leather tanning may show a much improved performance. With respect to changes anticipated in the relative ranking of these valves in the future, it should also be stated that the prosthetic group might well be the most likely to improve, owing to the almost limitless potential for development and application of new materials and designs.

In such an evaluation as this it is necessary to stand far enough away from the trees to see the outline of the forest. For this reason it is unwise to attempt to identify or credit each or any of the vast number of investigations and contributions which together comprise the body of knowledge relating to cardiac valvular replacement. However, this effort was prompted and most facilitated as a result of the author's opportunity to attend the First International Workshop on Tissue Valves, held near San Francisco, California, on 4-5 October 1969 and organized under the direction of Dr. Frank Gerbode. The participants, listed below, are pioneers in this field, and the impressions gained from their candid reports are the primary bases for this evaluation:

William W. Angell, M.D., Palo Alto, California. B. G. Barratt-Boyes, F.R.A.C.S., Auckland, New Zealand. H. H. Bentall, F.R.C.S., London, England. Jean Paul Binet, M.D., Paris, France. Alain Carpentier, M.D., Paris, France. W. P. Cleland, F.R.C.S., London, England. Eleanor M. K. Darby, Ph.D., Bethesda, Maryland. Charles DuBost, M.D., Paris, France. Sterling Edwards, M.D., Birmingham, Alabama. Frank Gerbode, M.D., San Francisco, California. Paul D. Harris, M.D., New York, New York. Raymond O. Heimbecker, M. D., Toronto, Ontario. Marion Ionescu, M.D., Leeds, England. Robert B. Karp, M.D., Birmingham, Alabama. C. Frederick Kittle, M.D., Chicago, Illinois. James R. Malm, M.D., New York, New York. Hitoshi Mohri, M.D., Seattle, Washington. Henry T. Nichols, M.D., Philadelphia, Pennsylvania. Mark F. O'Brien, F.R.C.S., Brisbane, Australia. Bruce C. Paton, F.R.C.S., Denver, Colorado. Gordon H. Puryear, M.D., Augusta, Georgia. Donald N. Ross, M.D., London, England. J. Keith Ross, F.R.C.S., London, England. Gudmon Semb, M.D., Oslo, Norway. Ake Senning, M.D., Zurich, Switzerland. Laurence Simpson, M.D., Durham, North Carolina. Alan S. Trimble, M.D., Toronto, Ontario. Clarence S. Weldon, M.D., St. Louis, Missouri. William P. Young, M.D., Madison, Wisconsin. 